\title{
Effects of social and emotional context on neural activation and synchrony during movie viewing
}

\author{
Sarah L. Dziura ${ }^{1}$, Junaid Merchant ${ }^{1}$, Diana Alkire ${ }^{1}$, Adnan Rashid ${ }^{2}$, Deena Shariq ${ }^{1}$, Dustin \\ Moraczewski $^{3}$, and Elizabeth Redcay ${ }^{1}$
}

1: The University of Maryland, College Park, MD

2: Georgetown University

3: National Institute of Mental Health

\begin{abstract}
Sharing emotional experiences impacts how we perceive and interact with the world, but the neural mechanisms that support this sharing are not well characterized. In this study, participants $(\mathrm{N}=52)$ watched videos in an MRI scanner in the presence of an unfamiliar peer. Videos varied in valence and social context (i.e., participants believed their partner was viewing the same (joint condition) or a different (solo condition) video). Reported togetherness increased during positive videos regardless of social condition, indicating that positive contexts may lessen the experience of being alone. Two analysis approaches were used to examine both sustained neural activity averaged over time and dynamic synchrony throughout the videos. Both approaches revealed clusters in the medial prefrontal cortex that were more responsive to the joint condition. We observed a timeaveraged social-emotion interaction in the ventromedial prefrontal cortex, although this region did not demonstrate synchrony effects. Alternatively, social-emotion interactions in the amygdala and superior temporal sulcus showed greater neural synchrony in the joint compared to solo conditions during positive videos, but the opposite pattern for negative videos. These findings suggest that positive stimuli may be more salient when experienced together, suggesting a mechanism for forming social bonds.
\end{abstract}

Keywords: social attention, social context, social neuroscience, affect

\section{Introduction}

Social experiences shape human behavior. Sharing the world with others is an integral part of how we perceive and learn about our world, as well as how we create social bonds ${ }^{1-3}$. The knowledge that we are attending to the same thing as another person (i.e., shared attention) can change our perception of that experience, and can impact cognitive processes such as memory, motivation, and judgments ${ }^{4-5}$. This shared attention often includes verbal or non-verbal communication among partners, but it does not necessarily require eye contact or even shared physical presence. Despite the importance of this phenomenon, open questions remain regarding how shared experiences affect the brain, individual attention, and behavior. The current study addresses these gaps by examining the neural, eye gaze, and behavioral responses to shared and non-shared contexts during naturalistic emotional video events.

Past work has primarily examined shared attention within a joint attention framework. Joint attention is the coordination of attention between two people onto a third, separate thing (for example, another person, an object, or an idea) at which point social partners achieve the state of shared attention awareness $^{6}$. These experiments often use simple cueing paradigms to study the separate steps that collectively make up joint attention, which ensure that attentional shifts are well-controlled ${ }^{7-9}$. This body of literature has pinpointed regions in the 
mentalizing network of the brain, including the posterior superior temporal sulcus and temporoparietal junction (pSTS/TPJ), dorsomedial prefrontal cortex (dmPFC), and posterior cingulate cortex (PCC), as critical for joint attention engagement ${ }^{6,10}$. However, it is not clear whether these regions are involved in the coordinating of attention through gaze or point cues, or the psychological state of shared attention itself.

While classic joint attention studies have the benefit of high stimulus control, they are limited by the passive, observational nature of the events. Reallife joint attention normally involves not only initiating or responding to visual social cues, but more complex interaction with actual social partners, including receiving feedback which allows for updating mental models of shared understanding and responding appropriately to continue the interaction or achieve a shared goal. Studies that have used interactive designs where participants believe they are responding to a partner in real time show more extensive engagement of both the medial and lateral regions of the mentalizing network, as well as some engagement of additional neural systems ${ }^{9,11-12}$. In particular, the reward network is engaged when anticipating and receiving feedback and is important for learning and decision-making ${ }^{13-14}$. Reward network activity may be prompted by expected responses from a joint attention partner (e.g., following pointing or gaze cues), although since these regions have been found in interactive paradigms, the engagement of this network could also be a product of the inherent rewarding nature of social stimuli itself, or else the addition of a live (or believed to be live) social partner ${ }^{9,12,15-16}$.

However, the psychological phenomenon of shared attention is difficult to examine even in interactive contexts within joint attention paradigms. Direct joint attention interactions with a partner include receiving visual gaze or pointing cues, which conflates the effect of visual attention with the perceptual experience of sharing attention.
Additionally, because interactions often include communicative feedback, these events may be confounded by the socially rewarding nature of interactions or more general processing related to predicting and receiving responses, as described in the previous paragraph. Examining how the brain supports sharing stimuli without direct communication can segregate feelings or awareness of social sharing, while controlling for these other perceptual, affective, and cognitive processes that arise during a direct social interaction. Due to these limitations, studying shared attention requires balancing the benefits of simulated realistic social experiences with the confounds that arise, as well as the restricted nature of the MRI scanner. Employing naturalistic stimuli in MRI designs, in particular dynamic viewing events over longer timescales, is one way to increase ecological validity ${ }^{17-18}$. Movie clips that simulate what the brain perceives in the real world engage a broad network of regions, are effective in inducing emotional states, and allow for social and non-social viewing designs without direct communication $^{19-22}$.

A final limitation of prior studies is that in past work shared events have mostly been neutral and often meaningless so that they were not complicated by effects of emotion. However, emotion and social context are tightly linked in the real world. Affiliative social behavior has been linked to a reduction in negative outcomes (such as stress responses) across a number of species including humans, a phenomenon known as social buffering ${ }^{23-}$ 24 . The mere presence of another person can cause a reduction in negative effects, even in the absence of affiliative behaviors or perceived support ${ }^{25-26}$. Social baseline theory hypothesizes that because the human brain has evolved within a highly social environment, its default state is to be surrounded by others ${ }^{27}$. Social groups have functioned throughout evolutionary history as a way to share burdens, diffuse or distribute responsibility, and protect against outside threats ${ }^{28-30}$. In fact, social deprivation or isolation causes abnormal stress levels, while 
social support acts as a buffer to reduce or normalize stress responses ${ }^{31-34}$. Similar effects also show up as lower BOLD activity in response to stressful stimuli when the brain is put in a social context compared to in isolation ${ }^{35-36}$.

Similar to social context impacting how we experience emotions, differing emotional content can in turn impact how we experience and respond to the presence of others. Emotions can be transferred socially, either through automatic mimicry or more explicit evaluation ${ }^{37-39}$. Collective gatherings in which participants perceive that they are in emotional synchrony with the group leads to both greater emotional reactions and stronger sociality ${ }^{40-}$ ${ }^{41}$. This body of literature indicates that social and emotional cognition are intertwined, but the relationship is likely context-dependent, and therefore difficult to disentangle. Additionally, these emotion and social context interactions have not typically been examined within the framework of shared attention.

There is some evidence that sharing emotional content with a partner may impact neural responses differently from non-emotional content. Viewing emotional pictures at the same time as a friend can increase positive feelings and subsequent activity in the reward network, irrespective of valence ${ }^{42}$. Direct feedback about emotional feelings from a partner during movie viewing has been shown to change one's own perception and increase neural coherence in the medial prefrontal cortex as well as regions of the emotional processing network ${ }^{21}$. Even viewing emotional videos alone can show individual differences in the mentalizing network (as well as emotion processing regions) that track with selfreported feelings of negative valence, which suggests a mechanism for promoting emotional sharing ${ }^{43}$. Together, these studies indicate that sharing emotional stimuli may cause greater engagement of regions within the mentalizing, emotional, and reward networks of the brain, although the question of how sharing dynamic positive and negative events without partner feedback impacts activity in any or all of these regions remains unsolved.

Shared attention in the real world is a sustained activity, yet naturalistic emotional content unfolds dynamically, with specific events causing greater or lesser emotional reactions. Thus, identifying these effects in the brain requires more recently developed analysis methods, as averaging over long periods of time can obscure or discount critical contextual details during tracking and responding to information. Inter-subject correlation (ISC) involves correlating time-locked activity across subjects to examine common patterns of neural processing ${ }^{44-45}$. This approach allows for a greater degree of specificity in tracking smaller fluctuations in the neural code and is therefore well suited to handle complex and dynamic stimuli ${ }^{21}$. On the other hand, traditional approaches such as grand averaging across discrete events (e.g., general linear models) can also provide important information about neural processing that is not captured by ISC. For example, primary auditory and visual areas of the brain show average signal increases to sounds and words within spoken and visual narratives, while more medial and lateral prefrontal regions respond to those same narratives over longer timescales ${ }^{46-47}$. To get a more complete picture of how the brain behaves in realworld contexts, it is important to look at and compare both time-averaged data to understand sustained response differences, and time-dependent data to understand dynamic or fluctuating responses.

The current study examined both neural and behavioral effects of sharing attention with a partner while viewing videos of varying emotional content. To examine neural effects, we collected functional MRI data during positive, neutral, and negative video events that participants either watched at the same time as a partner or alone. To examine behavior effects associated with these social and emotional contexts, we collected eye-tracking data and selfreported emotional and social feelings (i.e., perceived togetherness) about the viewing 
experience. We expected that perceived togetherness would increase during the joint condition compared to solo, and a corresponding difference in neural activity would be found within the mentalizing network of the brain. We also expected that perceived affect would change along with emotional video content; in particular, positive feelings would accompany more positive videos and negative feelings would accompany negative videos. Neurally, we hypothesized that videos with emotional content would recruit regions of the reward and emotional salience networks. We also expected to observe a social buffering effect on emotional feelings, with a boost in reported positive affect during the joint condition compared to solo. We expected that any observed emotion by social interactions in neural responses would occur within the aforementioned mentalizing, emotion, and reward networks.

\section{Methods}

The hypotheses and analysis plan for the data collected in this study were pre-registered on the Open Science Framework: https://osf.io/muzc3. Changes or amendments made to the planned methods and analyses are explained in each section.

\section{Participants}

52 participants (Mean age: 20.6; Age range: 1834; $\mathrm{F}=29, \mathrm{M}=23$; Race/Ethnicity: 43\% Asian, 16\% Black/African American, 6\% Hispanic/Latinx, 27\% White, and $8 \%$ Multiracial) were recruited from the undergraduate population at the University of Maryland. A total of 50 participants was sought, and our stopping rule was to collect usable data until all scheduled subjects were complete, which led to two additional participants. Data from an additional 3 subjects were collected but not included in analysis because they reported that they did not believe their confederate partner was a true participant. All participants were right-handed English speakers with normal or corrected-to-normal vision. All participants signed an informed consent form in accordance with the Declaration of Helsinki and the Institutional Review Board at the University of Maryland and were compensated for their time through money or course credit.

\section{Behavioral session}

The first session had participants get to know a peer through (1) an unstructured 5-minute conversation and (2) a 15-minute activity that they completed together where they came up with a common list of favorite things (either books, movies, TV shows, or music). This session aimed to induce familiarity among peers, as evidence suggests closeness increases effects of shared attention ${ }^{41}$ and past studies have examined shared attention in friend pairs ${ }^{42}$. The peer was not another participant, but actually an undergraduate research assistant confederate. We employed a total of 6 confederates throughout data collection, and gender matched confederates to participants. In this session the participants also completed surveys related to their social behavior and answered questions about their partner and the interaction they had.

\section{Imaging session}

Participants returned for a second session with their peer confederate at the Maryland Neuroimaging Center. The participants believed that they had signed up specifically for the MRI version of the experiment, while their partner (i.e., the confederate) had signed up for a behavioral version. They engaged in a brief practice session with their partner, where they watched either the same video or different videos on laptops. Both the practice and the experimental videos were presented in PsychoPy2 version 1.83.04 (Peirce et al., 2019). They were then brought into the scanner room, where their partner was directed to sit in front of a computer with a video 
camera trained on their profile. The participants were then set up in the scanner room.

During the session, participants viewed brief videos of positive, negative, and neutral affect content. These videos showed various scenarios involving humans, animals and the environment, lasting either 20 or 30 seconds. In half of the trials, participants believed they were watching the video at the same time as their partner, whereas in the other half of the trials they believed their partner was watching a different video. To control for potential confounds related to video content across social conditions, videos were counterbalanced so half of the subjects received a set of videos in the joint condition, and half of the subjects received the same video set in the solo condition. Participants rated their subjective feelings of aloneness vs. togetherness and positive vs. negative affect immediately after viewing, and then were shown what they believed to be either their partner's affect rating (in the joint condition) or the average affect rating from a separate group after watching the same video (in the solo condition). These ratings were identical across joint and solo conditions, and actually taken from average ratings for each video from a pilot group of subjects $(\mathrm{N}=20)$. The true data ranged from 2.6-7.6 and was re-categorized to fit the 1-9 scale and rounded to the nearest whole number. During each trial, participants also saw a smaller "live" video feed of their partner's profile next to the primary video that was randomly presented either on the left or the right side of the screen. These videos were pre-recorded and showed the confederates actually watching and reacting to the same videos that were used in the task (although they only showed the partner's face in profile and not the videos). In the joint condition, each video of the partner matched up with the main video shown to the participant, and the orientation of their face in the video was directed toward the screen. In the solo condition, the partner video was randomly selected from the pool of videos within emotional condition, meaning it showed them watching a different video, and the orientation of their face in the video was directed away from the screen. All of the partner videos used in the joint and solo conditions were identical over the course of the session to control for any condition-wide differential effects of facial expression or other visual content. See Supplementary Figure 1 for an example of this setup. Eye movements were tracked in the scanner with the EyeLink1000 system (SR Research; https://www.sr-research.com/). Sampling rate for this data was $1000 \mathrm{~Hz}$ and was collected from the right eye.

Functional MRI acquisition, pre-processing, and analysis

Functional MRI data were collected at the Maryland Neuroimaging Center on a 3.0 Tesla scanner with a 32-channel head coil (MAGNETOM Trio Tim System, Siemens Medical Solutions). Visual stimuli were presented on a rear projection screen and viewed by participants on a head coilmounted mirror.

A single T1 image was acquired using a threedimensional magnetization-prepared, rapid acquisition gradient-echo (MPRAGE) pulse sequence (192 contiguous sagittal slices, voxel size $=0.45 \times 0.45 \times 0.90 \mathrm{~mm}, \mathrm{TR}=1,900 \mathrm{~ms}, \mathrm{TE}=2.32$ $\mathrm{ms}$, flip angle $=9^{\circ}$, pixel matrix $=512 \times 512$ ), and opposite phase-encoding fieldmap scans (66 interleaved axial slices, voxel size $=2.19 \times 2.19 \times$ $2.20 \mathrm{~mm}, \mathrm{TR}=7,930 \mathrm{~ms}, \mathrm{TE}=73 \mathrm{~ms}$, flip angle $=$ $90^{\circ}$, pixel matrix $=96 \times 96$ ). Six runs of blood oxygenation level dependent (BOLD) task data were acquired using multiband-accelerated echo-planar imaging (66 interleaved axial slices, multiband factor $=6$, voxel size $=2.19 \times 2.19 \times 2.20 \mathrm{~mm}, \mathrm{TR}=1,250$ $\mathrm{ms}, \mathrm{TE}=39.4 \mathrm{~ms}$, flip angle $=90^{\circ}$, pixel matrix $=96$ $\times 96$ ). A further two runs of BOLD localizer data were acquired with the same parameters. These data were not used in the analyses described in this study. 
We utilized a publicly available standardized preprocessing pipeline (fMRIPrep 1.4.1 workflow ${ }^{49}$, available at 10.5281/zenodo.852659). Any differences in pre-processing steps from the preregistered planned analyses were due to this standardization of methods, which allows for more generalizable and potentially reproducible results. The following parameters were used to prepare data for analysis: The T1-weighted image was corrected for intensity non-uniformity, skull-stripped, and spatially normalized to standard space with a volume-based nonlinear registration. The BOLD runs were co-registered to the $\mathrm{T} 1 \mathrm{w}$ reference, slicetime corrected, and resampled into standard (MNI) space. Automatic removal of motion artifacts using independent component analysis (ICA-AROMA ${ }^{50}$ ) was performed on the preprocessed BOLD runs. Corresponding "non-aggressively" denoised runs were produced after such smoothing. A confounding time-series of framewise displacement (FD) was calculated based on the preprocessed BOLD. Full details of the parameters and programs used are found in the supplementary methods.

\section{General linear model}

A general linear model was conducted using AFNI's REMLfit program, with each of the six conditions of interest (joint positive, joint neutral, joint negative, solo positive, solo neutral, solo negative) and the 4-second rating period post-video collapsed across condition included in the model as regressors. These first-level results were then entered into a group-level mixed effects multilevel model using AFNI's 3dMVM. Planned analyses included a $2 \times 2$ model to examine main effects (joint vs. solo, emotion vs. neutral) and a social by emotion (i.e., positive + negative) interaction, as well as post-tests to examine individual contrast differences for each condition. We also conducted an exploratory $2 \times 3$ ANOVA to examine any main effect differences among the three emotion conditions, and social by emotional valence interaction effects. The betweensubject covariates included in these models were as follows: age, mean FD, gender-matched confederate, and counterbalanced group. Correction for multiple comparisons was conducted through calculating cluster sizes with probability thresholds above false positive (noise-only) clusters with AFNI's noise volume estimation and cluster simulation through spatial autocorrelation ("acf") functions. These cluster threshold values were calculated for each test separately and all significant data are presented at a voxel threshold of $p<0.001$ (bi-sided; $\mathrm{NN}=1$ ) with the associated minimum cluster threshold at $\alpha=0.05$ from these simulations (GLM: mentalizing mask = 12 voxels; emotion mask $=9$ voxels; reward mask $=$ 15 voxels / ISC: mentalizing mask $=12$ voxels; emotion mask $=10$ voxels; reward mask $=16$ voxels).

\section{Inter-subject correlation}

After each run was pre-processed through the fMRIprep pipeline, a general linear model regressed out the BOLD response from the rating periods after each video. The residuals from this analysis were then separated by individual video, with the onset and ending shifted by six volumes to accommodate the hemodynamic lag. These video responses were then re-concatenated into a consistent video order for every subject, separated by the six social $\mathrm{x}$ valence conditions (joint positive, joint neutral, joint negative, solo positive, solo neutral, solo negative). Because the videos were counterbalanced by social condition across subjects, the group average ISC was separated into two groups, and the analysis was conducted only within identical videos for each condition. Each subject's reordered time-series was correlated on a voxel-level with the average timeseries from the rest of their group, and the resulting $r$ values were transformed to subject-specific $\mathrm{z}$ maps.

These subject-level results were then entered into the same full multilevel model group analyses as the GLM to examine the (counterbalanced groupindependent) effects of social and emotional condition. These models included two within-subject 
variables of interest (social condition and emotional valence), as well as a number of nuisance variables: two categorical between-subject variables (group, confederate), and two quantitative between-subject variables (age, mean FD). Correction for multiple comparisons was again conducted through calculating cluster size thresholds with AFNI's clustsim function.

We aimed to replicate these group average ISC results by utilizing a crossed-random effects analysis method through pairwise ISC comparisons ${ }^{51-53}$. This approach accounts for the non-independence of the group due to each subject's data being represented $\mathrm{N}-1$ times in the model and has been shown to control the false positive rate (FPR) better than prior methods ${ }^{51}$. However, it has only been applied previously to linear mixed effects models with simpler A-B contrasts, rather than data with six conditions ( $2 \times 3$ setup). Due to the complexity of our model, we elected to fit our data within this framework by testing individual contrasts that were found to have significant results in the group average ISC approach to confirm their effect with this more conservative approach. Previous results using this method $^{53}$ have been shown to demonstrate a similar pattern but at a lower threshold of $p<0.01$. Similar to prior work, we found weaker effects in the same regions as the group average ISC results. These results are presented in the supplementary material.

\section{Networks of interest}

To test whether effects were found within hypothesized brain networks, we generated and downloaded network association maps from Neurosynth ${ }^{54}$ using the search terms "mentalizing", "emotion", and "reward" (thresholded at $\mathrm{Z}=4$, or $p$ $<0.0001$, two-tailed; Supplementary Figure 2). We used the Neurosynth mentalizing map instead of our own theory of mind functional localizer (as described in the pre-registration) to increase generalizability across populations and to ensure the maximum number of subjects were usable, as some did not have high quality localizer data. All $2 \times 2$ and $2 \times 3$ grouplevel analyses as well as significant cluster-size simulations were conducted within binarized masks generated from these three network maps. Some of these maps had regions that overlapped with each other, and therefore were used to constrain hypotheses rather than to make specific cognitive inferences about brain activity within one network compared to another.

\section{Significance testing}

F-test maps that reached a voxel threshold level of $p<0.001$ and cluster threshold of $\alpha<0.05$ were considered to be significant for main effects and interactions within each network of interest. The main social effect was tested within the mentalizing network, the emotion effect was tested within the emotion and reward networks, and the interaction was tested within all three networks. To probe interaction effects, post-test contrasts were conducted for each of the social effects within emotion conditions (joint emotions - solo emotions; joint neutral - solo neutral; joint positive - solo positive; joint negative - solo negative) and for each of the emotion effects within social conditions (joint emotions - joint neutral; solo emotions - solo neutral; joint positive - joint neutral; joint negative joint neutral; joint positive - joint negative; solo positive - solo neutral; solo negative - solo neutral; solo positive - solo negative). T-test maps that reached a voxel threshold level of $p<0.001$ and cluster threshold of $\alpha<0.05$ were considered to be significant for these contrasts. Contrast clusters that overlapped with interaction effect clusters are considered significant post-tests for these interactions, and are listed within the results tables in the overlapping contrast column. 


\section{Results}

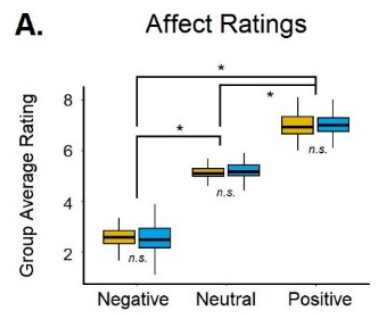

B. Togetherness Ratings

C. Time-Averaged Eye Tracking
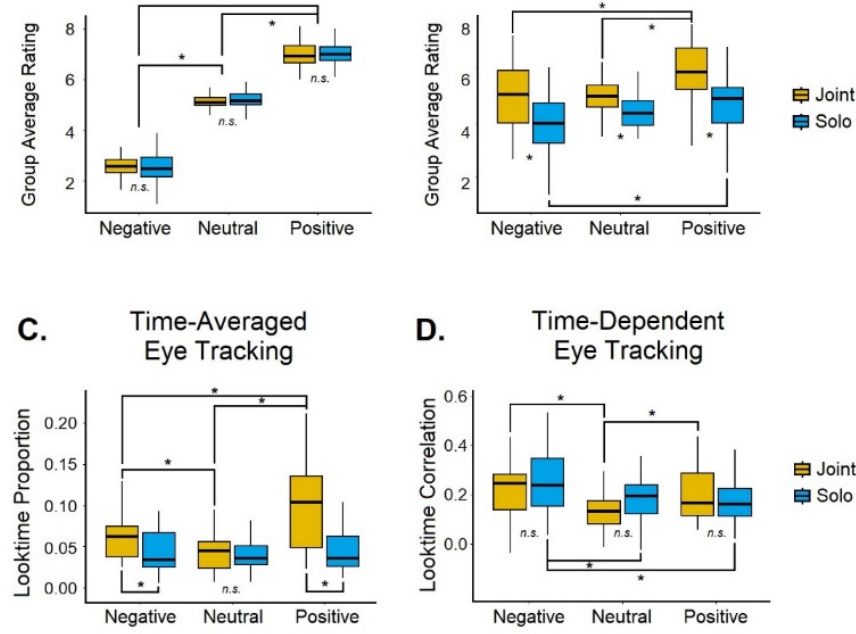

D. Time-Dependent

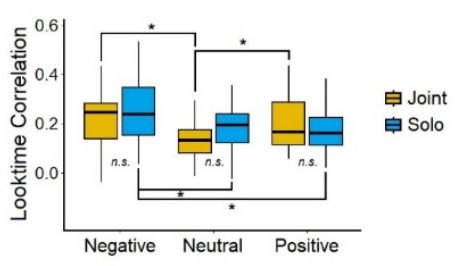

Emotion Condition

Figure 1. Behavioral Results. A. Group average $(\mathrm{N}=52)$ inscanner ratings of how emotional participants felt after watching each video on a scale of 0 (very negative) to 9 (very positive). B. Group average $(\mathrm{N}=38)$ in-scanner ratings of how alone or together they felt with their partner after watching each video on a scale of 0 (very alone) to 9 (very together). Fewer subjects are included in this data as the togetherness question was added after 14 scans had already been completed. C. Group average $(\mathrm{N}=27)$ of the proportion of time spent looking at the partner video feed compared to the main video feed. D. Group average $(\mathrm{N}=27)$ of the correlation between individual and group $(\mathrm{N}-$ subject) time spent looking at the confederate through the duration of each condition.

\section{Affect and togetherness ratings}

Affect ratings. As expected, participants reported feeling more negative while watching negative videos and more positive while watching positive videos $(\mathrm{F}(2,51)=472.8, p<0.0001$; Figure 1A). These results are consistent with a recent metaanalysis showing that movie clips are effective ways to induce positive and negative emotional states ${ }^{20}$. Contrary to our hypothesis, however, participants did not report feeling more emotional during the joint condition compared to solo $(\mathrm{F}(1,51)=0.005, p=$ $0.95)$. These null results do converge with recent work showing that shared video watching tasks do not meaningfully impact emotional ratings ${ }^{55}$.

Togetherness ratings. As expected, there was an effect of social condition wherein participants reported feeling more together with their partner during the joint condition compared to solo $(\mathrm{F}(1,37)$ $=21.2, p<0.0001$; Figure 1B). There was also a main effect of emotion $(\mathrm{F}(2,37)=18.3, p<0.0001)$ and a social-emotion interaction $(\mathrm{F}(1,37)=3.61, p<$ 0.05 ) in togetherness ratings, showing that participants felt less alone during positive videos compared to both negative and neutral, and that the strongest feelings of togetherness were in the positive joint condition, and the strongest feelings of aloneness were in the negative solo condition.

\section{Eye tracking}

We also examined eye tracking data that was collected during the scan. Twenty-seven out of the 52 fMRI subjects had usable data for analysis. In order to determine differences between individual visual attention behaviors and synchronous visual attention behaviors, we examined the data in two different ways: time-averaged and time-dependent. This also allows for more analogous comparison between these behavioral data and fMRI results. Time-averaged (analogous to the GLM fMRI method) was calculated as the proportion of time spent looking at the partner feed compared to the main video across each entire condition. Timedependent results, in contrast, are analogous to the ISC fMRI method. Each subject's time spent looking at the partner video (data sampled at $1250 \mathrm{~ms}$, equivalent to 1TR) was concatenated for all videos within each condition. We then calculated the correlation between the subject and the group average without the subject (leave-one-out) and entered these correlation results into a full group model.

Time-averaged. We found that overall, people looked more at their partner during the joint condition compared to solo conditions $(\mathrm{F}(1,25)=$ $22.12, p<0.001$; Figure 1C), and that there was an emotion by social interaction such that emotional conditions showed a greater proportion of partner looking compared to neutral $(\mathrm{F}(1,25)=11.8, p<$ 
0.001). Post-tests revealed a greater amount of partner looking during joint positive compared to joint negative $(\mathrm{t}=3.4, p<0.001)$, and joint positive compared to solo positive $(\mathrm{t}=5.687, p<0.0001)$, indicating a bias to look towards one's partner with positive emotional content during social experiences).

Time-dependent. On the other hand, there was no social effect within the time-dependent results, but there was a significant main effect of emotion $(\mathrm{F}(1,25)=5.1, p<0.05$; Figure 1D). Follow-up ttests showed that emotional videos caused more consistency in when participants looked at their partner compared to neutral $(t=3.7, p=0.001)$. This was particularly driven by negative videos, which elicited more consistency than either neutral or positive $(\mathrm{t}=4.15, p<0.001 / \mathrm{t}=2.24, p<0.05)$.

Taken together, the time-averaged and timedependent eye-tracking results suggest that differing emotional valence is an important signal in social sharing, where people may more often seek out or orient toward a social partner during positive emotional times, but there is little consistency in when this happens during the content.

\section{A. Social Main Effect: Mentalizing Mask}
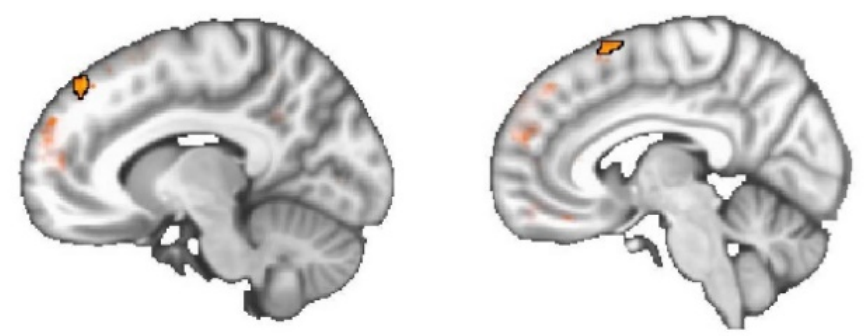

\section{B. Emotion Main Effect: Emotion Mask}
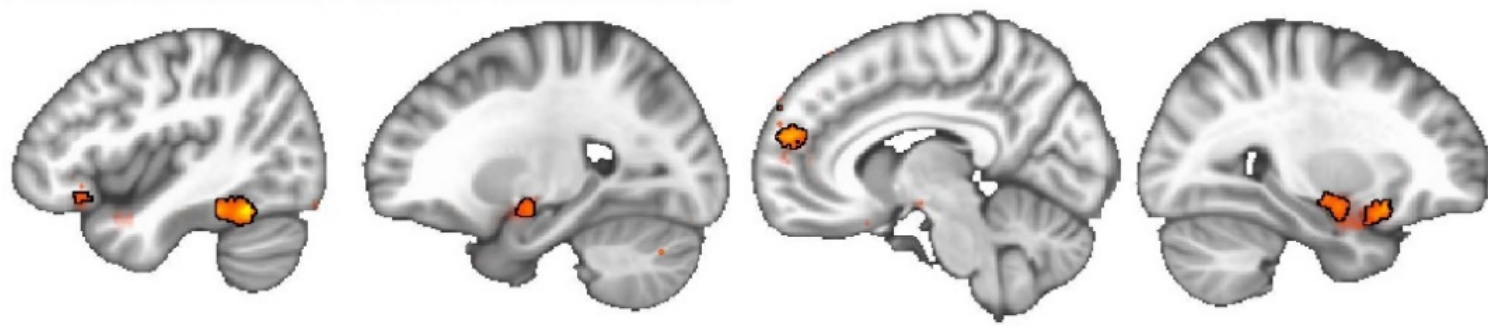

\section{Emotion Main Effect: Reward Mask}
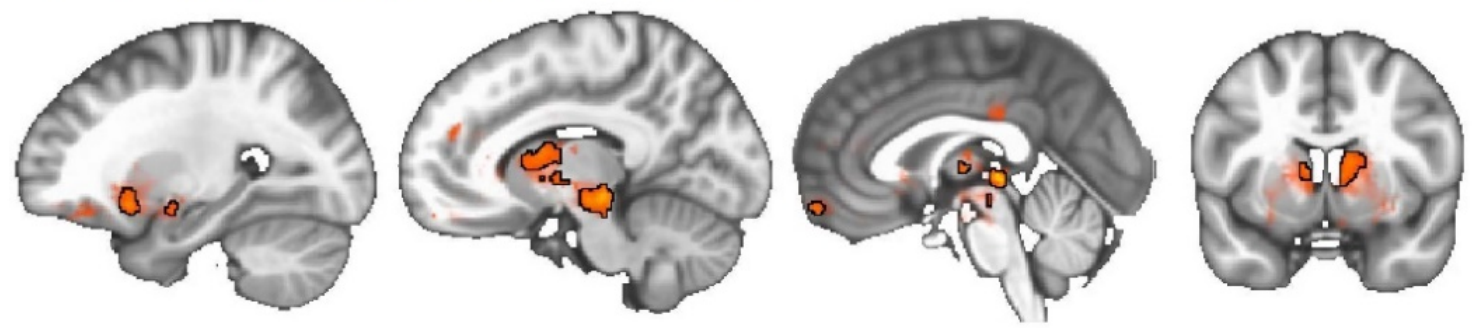

$$
F=0
$$$$
F=99.9
$$

Figure 2. ANOVA main effect result maps from the time-averaged data. Significant clusters are outlined in black at a voxel threshold of $p<0.001$ (cluster $\alpha=0.05$ ). Sub-threshold effects (i.e., F $>0, p>0.001$ ) are displayed with transparent fade. A. Social main effect within the mentalizing network. B. Emotion main effect within the emotion network. C. Emotion main effect within the reward network. 
Table 1. Time-Averaged Social Main Effect.

\begin{tabular}{lccccccc}
\hline \hline \multicolumn{1}{c}{ Region } & X & Y & Z & Cluster Size & Peak F & Contrast & t-value \\
\hline \hline R Superior Frontal Gyrus & 6 & 16 & 64 & 21 & 21.19 & Joint > Solo & 4.33 \\
L Dorsomedial Prefrontal Cortex & -10 & 42 & 48 & 12 & 23.47 & Joint $>$ Solo & 4.84 \\
\hline \hline
\end{tabular}

Table 2: Time-Averaged Emotion Main Effect $(2 \times 2)$.

\begin{tabular}{|c|c|c|c|c|c|c|c|c|}
\hline Mask & Region & $\mathbf{X}$ & $\mathbf{Y}$ & $\mathbf{Z}$ & Cluster Size & Peak F & Contrast & $t$-value \\
\hline \multirow{13}{*}{ Emotion } & R Amygdala & 22 & -6 & -10 & 186 & 41.03 & Emotion $>$ Neutral & 5.93 \\
\hline & R Hippocampus & 28 & 18 & -14 & 162 & 47.54 & Emotion $>$ Neutral & 6.9 \\
\hline & L Temporal Fusiform Cortex & -42 & -54 & -18 & 116 & 70.59 & Emotion $>$ Neutral & 8.4 \\
\hline & L Amygdala & -20 & -8 & -12 & 93 & 35.11 & Emotion $>$ Neutral & 5.93 \\
\hline & Brain Stem & 4 & -30 & -2 & 68 & 58.24 & Emotion $>$ Neutral & 7.63 \\
\hline & R Medial Prefrontal Cortex & 8 & 56 & 20 & 62 & 45.09 & Emotion $>$ Neutral & 6.72 \\
\hline & R Medial Prefrontal Cortex & 4 & 56 & 34 & 49 & 40.5 & Emotion $>$ Neutral & 6.36 \\
\hline & L Temporal Pole & -36 & 14 & -26 & 17 & 28.65 & Emotion $>$ Neutral & 5.35 \\
\hline & L Ventrolateral Prefrontal Cortex & -34 & 24 & -16 & 13 & 22.35 & Emotion $>$ Neutral & 4.73 \\
\hline & L Ventrolateral Prefrontal Cortex & -42 & 26 & -12 & 12 & 19.09 & Emotion $>$ Neutral & 4.37 \\
\hline & L Medial Prefrontal Cortex & -8 & 52 & 18 & 10 & 20.29 & Emotion $>$ Neutral & 4.5 \\
\hline & R Ventrolateral Prefrontal Cortex & 54 & 32 & -8 & 9 & 40.06 & Emotion $>$ Neutral & 6.33 \\
\hline & R Medial Prefrontal Cortex & 18 & 58 & 18 & 9 & 17.45 & Emotion $>$ Neutral & 4.18 \\
\hline \multirow{6}{*}{ Reward } & Brain Stem & 4 & -30 & -2 & 601 & 58.2 & Emotion $>$ Neutral & 7.63 \\
\hline & $\mathrm{R}$ Thalamus & 8 & -2 & 8 & 175 & 28.11 & Emotion $>$ Neutral & 5.3 \\
\hline & L Ventrolateral Prefrontal Cortex & -26 & 14 & -12 & 137 & 31.29 & Emotion $>$ Neutral & 5.59 \\
\hline & L Caudate & -8 & 4 & 12 & 86 & 30.24 & Emotion $>$ Neutral & 5.5 \\
\hline & L Amygdala & -20 & -4 & -12 & 30 & 23.97 & Emotion $>$ Neutral & 4.9 \\
\hline & Ventromedial Prefrontal Cortex & -2 & 58 & -18 & 27 & 31.78 & Emotion $>$ Neutral & 5.6 \\
\hline
\end{tabular}


In contrast, negative videos seem to elicit more content-dependent gaze shifts between videos, as there were no social effects found in the consistency over time measure. Post-test results showed that solo negative videos elicited more consistency than either neutral or positive, whereas both conditions of joint emotional videos were greater than joint neutral, and not significantly different from each other.

\section{Functional MRI}

We conducted several multivariate model analyses to test both confirmatory and exploratory questions. Confirmatory analyses were done through $2 \times 2$ group-level tests within hypothesized brain networks to examine the main effects of social context, valence-collapsed emotion (vs. neutral) content, and the interaction between these. We further explored whether there were differential interactions due to valence by conducting $2 \times 3$ test (emotions = positive, neutral, and negative). All tests were run on both time-averaged (GLM) and time-dependent (ISC) data.

\section{Time-averaged}

To test whether time-averaged effects were present in the hypothesized mentalizing, emotion, and reward brain networks, we used three separate Neurosynth-generated masks to restrict our analyses.

Social. As expected, the GLM analysis revealed a main effect of social condition (joint $>$ solo) in the mentalizing network, specifically the dorsomedial prefrontal cortex (dmPFC) (Figure 2A; Table 1).

Emotion. A main effect of emotion content (emotions $>$ neutral) was observed in both the emotion and reward networks (Figure 2B-C; Table 2). There was a large overlap in main effect activity patterns between the valencecollapsed 2 × 2 (emotions, neutral) and valenceseparated $2 \times 3$ (positive, neutral, negative) models (see Supplementary Table 1 for $2 \times 3$ results maps).

Interaction. An interaction between social and emotion conditions was observed within a ventromedial prefrontal cortex cluster of the brain which was present in both mentalizing and reward network masks (Figure 3A; Table 3). This same region was found to be significant in both valence-collapsed and valence-separated models (Table 3). Overlapping post-test contrasts showed that the valence-collapsed cluster was more responsive to joint emotion than solo emotion or joint neutral videos, showing a sensitivity to social sharing of emotions in particular (Figure 3B; Table 3). The valence separated model indicated that the joint vs. solo emotion effect found is driven particularly by negative emotions, as this region was more sensitive to joint negative than solo negative videos only. This model also indicated a greater responsivity to both solo positive and neutral than solo negative videos, showing a sensitivity to differential emotions even in the absence of social sharing (Figure 3C; Table 3). 


\section{Interaction Effect: Social x Emotion}

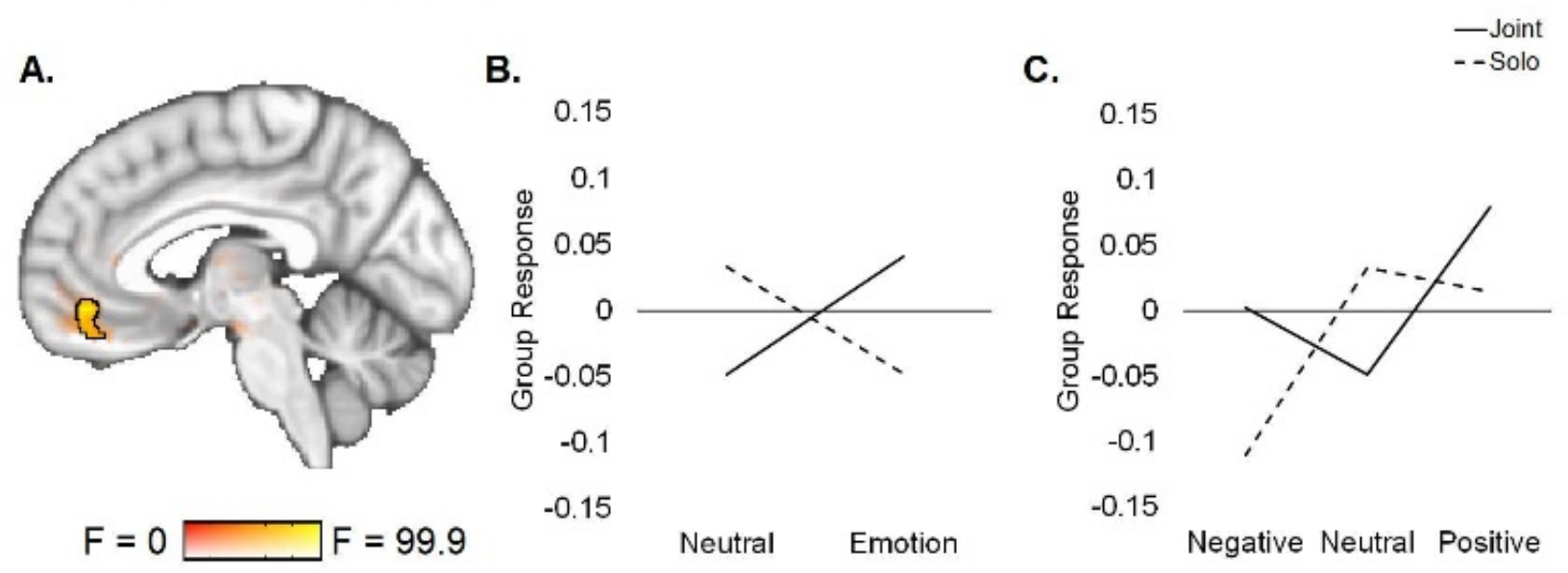

Figure 3. ANOVA social-emotion interaction results from the time-averaged data. Significant cluster is outlined in black at a voxel threshold of $p<0.001$ (cluster $\alpha=0.05$ ). Sub-threshold effects (i.e., $\mathrm{F}>0, p>0.001$ ) are displayed with transparent fade and not outlined. Line graphs plot extracted values from each relevant condition to illustrate direction of significant effects. A. The same vmPFC region located within both mentalizing and reward masks was found to be significant in the $2 \times 2$ and $2 \times 3$ models, although the cluster size and peak voxel location differed by model $(2 \times 2$ reward mask results shown: Table 3 shows all cluster information). B. Extracted values for from the $2 \times 2$ (emotion, neutral) model in the reward mask. C. Extracted values from the $2 \times 3$ (positive, neutral, negative) model in the reward mask.

Table 3. Time-Averaged Interaction Effects.

\begin{tabular}{|c|c|c|c|c|c|c|c|c|}
\hline Mask & Region & $\mathbf{X}$ & $\mathbf{Y}$ & $\mathbf{Z}$ & $\begin{array}{l}\text { Cluster } \\
\text { Size }\end{array}$ & Peak F & Overlapping Contrast & $t$-value \\
\hline \multicolumn{9}{|c|}{ Interaction: 2 x 2} \\
\hline Reward & Ventromedial Prefrontal Cortex & -4 & 46 & -10 & 57 & 22.81 & $\begin{array}{l}\text { Emotions: Joint }>\text { Solo } \\
\text { Joint: Emotion }>\text { Neutral }\end{array}$ & $\begin{array}{l}4.53 \\
3.63\end{array}$ \\
\hline Mentalizing & Ventromedial Prefrontal Cortex & -2 & 44 & -12 & 15 & 15.26 & Emotions: Joint $>$ Solo & 4.48 \\
\hline \multicolumn{9}{|c|}{ Interaction: 2 × 3} \\
\hline Reward & Ventromedial Prefrontal Cortex & -4 & 46 & -10 & 48 & 15.23 & $\begin{array}{l}\text { Negative: Joint }>\text { Solo } \\
\text { Solo: Neutral }>\text { Negative } \\
\text { Solo: } \text { Positive }>\text { Negative }\end{array}$ & $\begin{array}{l}4.86 \\
4.53 \\
4.89\end{array}$ \\
\hline Mentalizing & Ventromedial Prefrontal Cortex & -2 & 44 & -12 & 17 & 9.8 & & \\
\hline
\end{tabular}


A. Social Main Effect: Mentalizing Mask

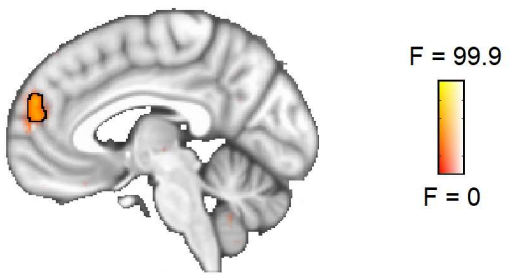

B. Emotion Main Effect: Emotion Mask
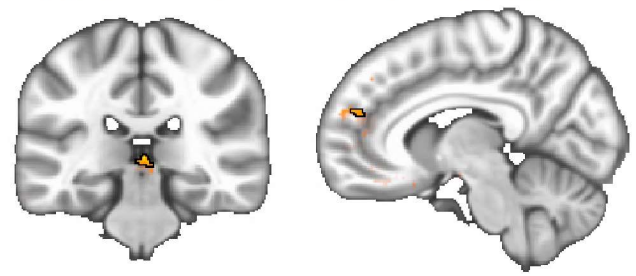

C. Emotion Main Effect: Reward Mask
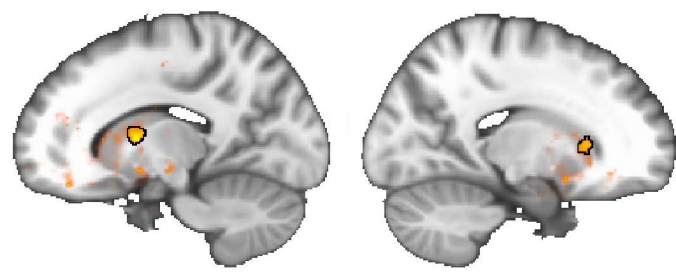

Figure 4. ANOVA results from the time-dependent (ISC) data. Significant clusters are outlined in black at a voxel threshold of $\mathrm{p}<0.001$ (cluster $\alpha=0.05$ ). Sub-threshold effects (i.e., F $>0, p$ $>0.001$ ) are displayed with transparent fade and not outlined.

A. Main social effect within the mentalizing network. B. Main $2 \times 3$ emotion (positive, neutral, negative) effect within the emotion network. C. Main 2 × 3 emotion (positive, neutral, negative) effect within the reward network.

\section{Time-dependent}

To test whether time-dependent effects were present in the hypothesized mentalizing, emotion, and reward brain networks, we used the same masks to restrict our ISC analyses.

Social. The time-dependent (ISC) analysis revealed a main effect of social condition (joint $>$ solo) in the dorsomedial PFC (Figure 4A; Table 4).
Emotion. The valence-collapsed model did not show any significant main effect of emotion within either the emotion or reward networks. However, small clusters were observed in the valenceseparated model in both of these networks (Figure 4B-C; Table 5), indicating that positive and negative videos are tracked differently over time within these networks, even though the time-averaged results show that similar regions process both positive and negative emotions overall.

Interaction. Social-emotion interaction clusters were found in the mentalizing network in both models (left anterior temporal lobe (ATL): Figure 5A; left STS: Figure 5B; Table 6) and in the emotion network in the valence-separated model only (bilateral amygdalae: Figure 5C; Table 6). Overlapping post-test individual contrasts in the bilateral amygdalae show greater synchrony for joint positive than solo positive events, but a trending opposite pattern for negative events, where solo is greater than joint $(p<0.005)$. The individual contrasts also show greater synchrony for joint positive than joint negative events. The left ATL post-tests indicate that the interaction effect in this region is driven by the neutral condition (joint $>$ solo), and the left STS post-tests indicated that the interaction effect in this region is driven by negative events (negative $>$ neutral in solo condition only).

\section{Exploratory analyses}

Additional analyses were conducted to confirm our results and to provide hypotheses for further study. We examined whether other brain regions outside the three hypothesized networks supported dynamic social-emotion processing through wholebrain (non-mask-restricted) analyses. Many of the regions found for the main effects were the same as 
within the network-restricted analyses, although we also observed that the main emotion effect in the time-dependent analysis engaged a much broader extent of the cortex (Supplementary Figure 7B-C). There were no additional social main effects in the time-averaged analysis, although a cluster in the cerebellum was observed in the time-dependent analysis (Supplementary Figure 7A). Both the time-averaged and time-dependent results also yielded several additional significant interaction clusters in visual and fronto-parietal regions of the brain (Supplementary Figures 6 \& 8), suggesting differential effects of attention and eye gaze. Neural synchrony was found to be increased to solo emotional content and shared neutral content within these, indicating that attentional shifts are more similar across participants when stimuli have a singular salient dimension (e.g. either emotion content or social context) rather than the inclusion or absence of both. Full details are included in the supplementary text.

Table 4. Time-Dependent Social Main Effect.

\begin{tabular}{cccccccc}
\hline \hline Region & $\mathbf{X}$ & $\mathbf{Y}$ & $\mathbf{Z}$ & Cluster Size & Peak F & Contrast & t-value \\
\hline \hline Medial Prefrontal Cortex & -6 & 56 & 22 & 85 & 22.31 & Joint $>$ Solo & 4.83 \\
\hline \hline
\end{tabular}

Table 5. Time-Dependent Emotion Main Effect (2 x 3).

\begin{tabular}{llccccccc}
\hline \hline Mask & \multicolumn{1}{c}{ Region } & $\mathbf{X}$ & $\mathbf{Y}$ & $\mathbf{Z}$ & Cluster Size & Peak F & Overlapping Contrast & $\boldsymbol{t}$-value \\
\hline \hline \multirow{2}{*}{ Emotion } & Brain Stem & 2 & -30 & 2 & 26 & 15.83 & Negative $>$ Neutral & 5.83 \\
& Medial Prefrontal Cortex & 20 & -10 & -12 & 10 & 12.05 & & \\
\hline \hline \multirow{2}{*}{ Reward } & L Putamen & -14 & 2 & -8 & 36 & 17.28 & Neutral > Positive & 3.99 \\
& & & & & & & Negative $>$ Positive & 5.54 \\
& R Caudate & 18 & 22 & -6 & 23 & 12.26 & Negative $>$ Positive & 4.09 \\
& Brain Stem & 6 & -26 & 4 & 18 & 15.1 & $\begin{array}{c}\text { Negative }>\text { Neutral } \\
\text { Negative }>\text { Positive }\end{array}$ & 3.79 \\
& & & & & & & & \\
\hline \hline
\end{tabular}

Table 6. Time-Dependent Interaction Effects.

\begin{tabular}{|c|c|c|c|c|c|c|c|}
\hline Region & $\mathbf{X}$ & $\mathbf{Y}$ & $\mathbf{Z}$ & Cluster Size & Peak F & Overlapping Contrast & $t$-value \\
\hline \multicolumn{8}{|l|}{ Interaction: 2 x 2} \\
\hline L Anterior Temporal Lobe & -56 & -6 & -24 & 15 & 26.67 & Neutral: Joint > Solo & 4.54 \\
\hline \multicolumn{8}{|l|}{ Interaction: 2 x 3} \\
\hline R Amygdala & 26 & -10 & -16 & 60 & 21.41 & $\begin{array}{c}\text { Positive: Joint }>\text { Solo } \\
\text { Joint: Positive }>\text { Neutral } \\
\text { Joint: Positive }>\text { Negative }\end{array}$ & $\begin{array}{l}3.92 \\
3.89 \\
4.84\end{array}$ \\
\hline
\end{tabular}




\section{A. Emotion (2) x Social Interaction: Mentalizing Mask}

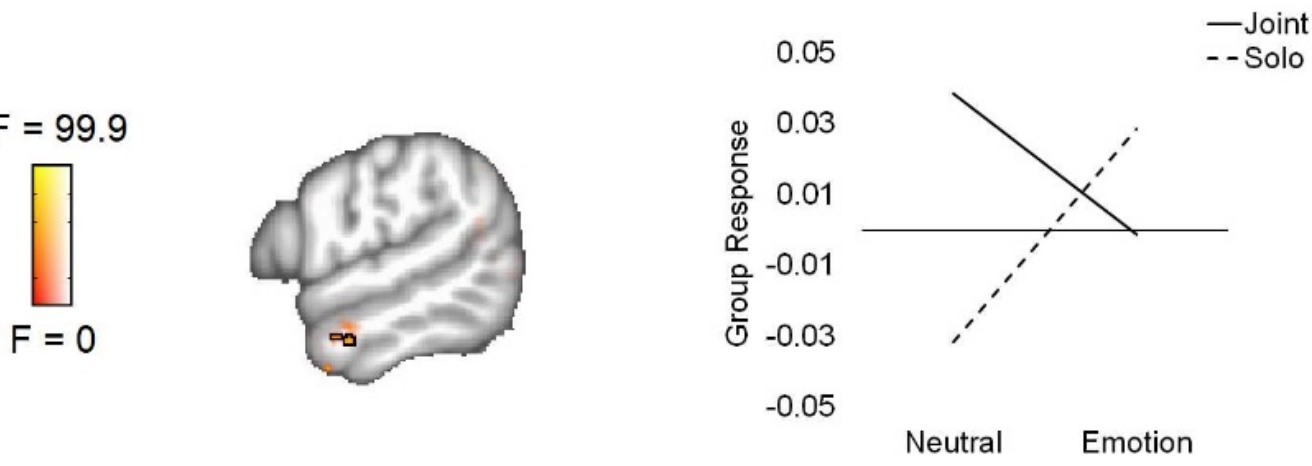

B. Emotion (3) x Social Interaction: Mentalizing Mask
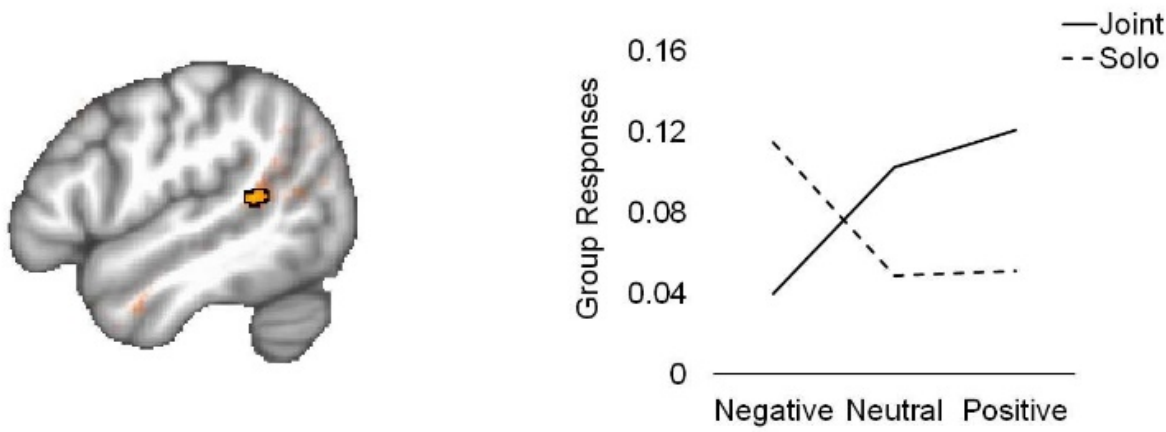

\section{Emotion (3) x Social Interaction: Emotion Mask}
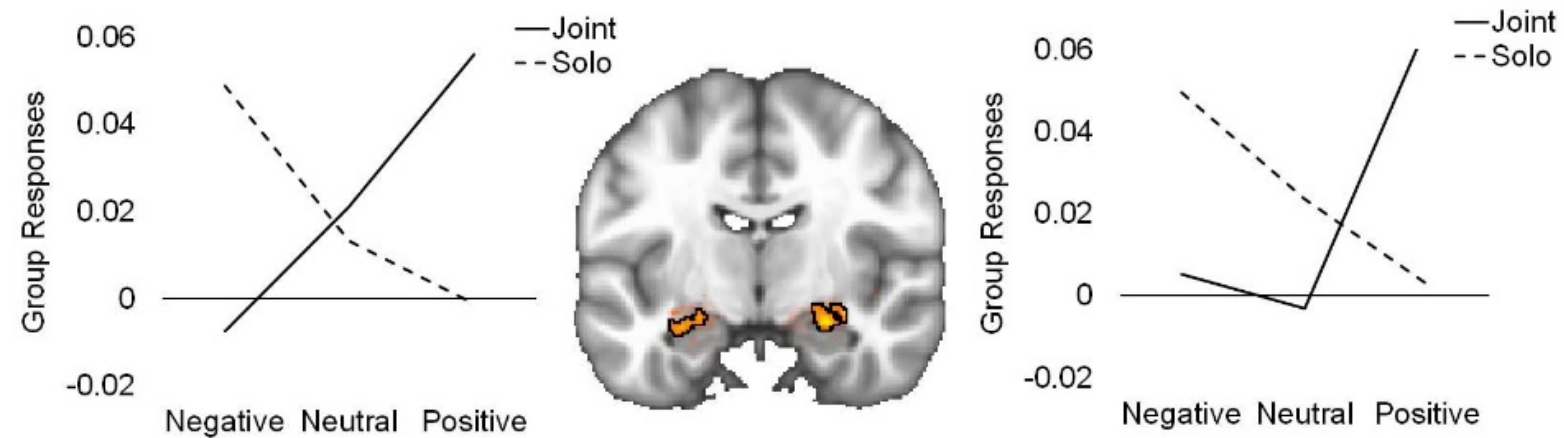

Figure 5. ANOVA social-emotion interaction results from the time-dependent (ISC) data. All group tests were conducted within three separate masks generated from Neurosynth and reflecting different brain networks: mentalizing, emotion, and reward. Significant cluster is outlined in black at a voxel threshold of $p<0.001$ (cluster $\alpha=0.05$ ). Sub-threshold effects (i.e., $\mathrm{F}>0, p>0.001)$ are displayed with transparent fade and not outlined. Line graphs plot extracted values from each relevant condition to illustrate direction of significant effects. A. Interaction from the 2 x 2 valence-combined (emotion, neutral) model found in the mentalizing network. B. Interaction from the 2 x 3 valence-separated (positive, neutral, negative) model found in the mentalizing network. C. Interaction from the $2 \times 3$ valenceseparated model found in the reward network. 


\section{Discussion}

This study examined the neural and behavioral effects of social and emotional context through naturalistic viewing of emotional videos both with a partner and alone. We found evidence that the medial prefrontal cortex (mPFC) supports social sharing through both sustained and temporally fluctuating activity. We also found that the ventromedial prefrontal cortex (vmPFC) is sensitive only to sustained social-emotional shared events, whereas the amygdala and areas of the left superior temporal sulcus track responses across the duration of the shared emotional events. Within these timedependent results, we observed a valence dissociation between positive content with a partner and negative content when alone, indicating that the brain takes social context into account when viewing different types of emotion-eliciting events, and that the salience of these events may be affected by the presence or absence of another person.

We found converging evidence through two methods of analysis that clusters within the mPFC are engaged in social event sharing, irrespective of the emotional content of that experience, on both a time-averaged (i.e., sustained responses over the duration of video events) and time-dependent (i.e., allowing for dynamic shifts during the video events) scale. The mPFC has been found to be involved in responding to joint attention without interaction (i.e., recorded gaze cues) ${ }^{56}$ as well as both initiating and responding to joint attention cues from a computer avatar $^{9,57}$ and a live partner (Redcay et al., 2012). The $\mathrm{mPFC}$ activity found in this study could be linked to similar gaze-directed behavior, as we also observed that participants looked toward the video of their partner more on average during shared events. Although studies have found mPFC associations with both initiating and responding to joint attention ${ }^{9,58}$, it is suggested to be a particularly important part of the goal-directed attention system (in contrast to the orienting and perceptual attention system), and therefore supports higher-order related processes such as intent to communicate with a partner ${ }^{58-60}$. In our case the partners were not looking back (and not expected to) so the effect was not directly interactive, but it may still indicate the desire to share with a partner prior to interaction. However, partner referencing is not sufficient to explain all mPFC activity, in particular the time-dependent social effect, as we did not see corresponding timedependent social eye-tracking effects. This suggests that certain events in the videos elicited partnerrelated processing but did not cause gaze shifts. The mPFC has been suggested to play a role in personspecific mentalizing as well as self-referential and introspective thought more generally ${ }^{61-62}$, and therefore likely involves making connections between these as well as reasoning about triadic associations (e.g., me, you, and other) ${ }^{63}$. Our data show that this same region responds to shared attention events even in the absence of passive or interactive cues, suggesting that activity is driven by the self-generated phenomena of a shared experience, rather than any external social input from a partner.

We also observed that the medial and dorsomedial areas of the PFC that were engaged in social sharing overall are not sensitive to interactions with emotion; instead, it is the ventromedial region of the PFC that responds differently to these conditions. In particular, the vmPFC shows a greater sustained response for social-emotional events compared to non-social, non-emotional (i.e. neutral alone condition) events. We also observed that it is sensitive to emotional differences within social conditions. This region was identified within both mentalizing and reward network analyses, which is consistent with its placement in an extended socioaffective-default network characterized through conjunction and connectivity meta-analyses ${ }^{61,64}$. As it is densely connected to the ventral striatum, it is thought to support processes of reward-based learning and feedback in both social and non-social contexts $^{65-67}$, as well as motivation and cognitive modulation of affect ${ }^{64}$. The time-averaged results 
presented here converge with several decades of research showing a dissociation between dorsal/medial and ventromedial areas of the PFC along similar lines ${ }^{65,68-70}$. However, we found that the vmPFC does not dynamically track these different conditions, as this region did not show a significant interaction in the ISC results. This is consistent with prior work that shows an association with periods of self-generated thought ${ }^{71}$, which are more heterogeneous and therefore likely not sensitive to synchronous fluctuations in neural activity across people.

In contrast, we found a time-dependent interaction in the bilateral amygdala, as well as smaller clusters in the left temporal lobe (pSTS and anterior temporal lobe). The amygdala in particular showed more synchronous activity to joint positive compared to joint neutral and negative as well as solo positive events, as well as a trending opposite effect (solo negative $>$ joint negative). The amygdala has been demonstrated to be sensitive to personally relevant stimuli, including both social and emotional content $^{72-73}$. These interaction results suggest that negative events may be more salient without social context (or else are unaffected by social context), whereas positive events are far more salient when shared with a social partner. The region has also been strongly linked to threat detection and responses ${ }^{74-75}$, and threatening environments may prove more hostile and important to attend to when alone, due to an inability to distribute the burden among a group. On the other side, positive events could be a specific driver of socially relevant behavior. This is consistent with findings that reward network activity in response to positive events (such as winning money) is modulated by social presence, interaction, and cooperation ${ }^{76-78}$. Our findings indicate that this modulation does not require explicit rewarding feedback (e.g., money, or a smiling face), and therefore may be a consequence of the shared attention itself.
Our behavioral data in the form of eye tracking and togetherness ratings also indicate a particular relevance of positive content during shared events. We observed relatively greater feelings of togetherness with a partner during the positive condition compared to neutral or negative, and participants also spent a proportionally greater amount of time looking at their partner during the positive condition compared to neutral and negative. Even in the absence of the social partner, positive videos increased ratings of togetherness, which suggests that the experience of being alone is less salient during these times, in contrast to neutral trials (which may be boring) or negative trials (which may be upsetting). One limitation of using these types of naturalistic events is that there could still be some effects of video content unrelated to emotional feelings induced, although we attempted to control for some of this (e.g., the same number of visible human faces across positive and negative video conditions). However, we observed even greater increases in look time and togetherness during the joint positive compared to solo positive condition, even though these conditions contained identical videos. This shows that our results cannot simply be explained by video content, and suggests that positive content doesn't just yield the absence of feelings of aloneness, but may induce a drive or desire to share positive events with others. There are several potential outcomes for this behavior. Evidence suggests that positive emotions are linked to greater well-being ${ }^{79}$ and that sharing positive experiences with others leads to greater resilience ${ }^{80}$, so there may be psychological benefits to the individual engaging in sharing behavior. Additionally, sharing experiences can lead to increased willingness to engage with a stranger and greater social bonding in both adults and children $^{3,55,81-82}$, so this may also serve as a way to form or strengthen social relationships among the pair.

Both the reward and emotion networks showed an expected main effect of emotion in the time-averaged 
analysis, with greater activation in many regions to emotional compared to neutral videos. However, the time-dependent analysis did not show much of an emotion effect within either of these masks. The valence-separated model showed some significant differences within the brain stem, dorsal striatum, and $\mathrm{mPFC}$, and overlapping contrasts revealed this is due to greater synchrony for negative videos in nearly every cluster. These central regions of the brain support affective and motor processing and have been linked to integrating motivation and action $^{83-84}$. As we also observed greater eye-tracking consistency in when participants looked at their partner during negative videos than either neutral or positive in both joint and solo social conditions, it is possible that these regions are driving this emotionrelated looking behavior. This does not explain why participants looked more at their partner during negative events even in solo trials, but we can only speculate on potential reasons. It may be that the negative videos more often included singular emotional events compared to positive and neutral videos, so there was greater consistency in looking behavior throughout the videos. The negative events also included some upsetting material, so participants may have been motivated to look away from the main video rather than look toward their partner specifically. More research is needed to determine the causes of motivated emotional looking behavior.

Comparing ISC and GLM outputs from identical data allows us to build upon findings from classic literature to better explain how the brain functions in naturalistic settings. GLM models show sustained activity differences across entire events, while ISC shows which regions of the brain have reliable responses during dynamic events irrespective of response amplitude ${ }^{85}$. The results presented in this paper confirm and extend our understanding of how social-emotional processing unfolds in the brain during shared attention situations. It is well established that regions of the brain accumulate information over time along a hierarchy of receptive windows ${ }^{86-87}$. Regions such as primary sensory cortices are receptive to shorter spatial and temporal windows, allowing for greater detail and specificity of relevant inputs ${ }^{88-89}$. Higher order regions are more receptive to longer timescales, allowing for the integration of memory and contextual information as sensory input accumulates ${ }^{90-91}$. Greater specialization in areas that support longer temporal receptive windows is associated with cortical maturity and greater comprehension of scenarios involving social information ${ }^{53}$. Our results show that the bilateral amygdalae and areas of the temporal lobe are receptive to shorter and more dynamic fluctuations in social-emotional content and context. In contrast, we found that a more anterior region of the brain (vmPFC) produces sustained activity that is sensitive to social-emotional differences in content and context over a longer period of time (20 and 30 second videos).

In conclusion, this study examined time-averaged and time-dependent neural processing of naturalistic social-emotional scenes with a partner and alone. We show there are context-dependent differences in positive and negative content that lead to differential attentional allocation and feelings of sociality. We also show some similarities and some differences in which brain areas and networks respond maximally to different conditions and what regions track subject-consistent fluctuations for the duration of the viewing. These results help shed light on the complex interactions between social and emotional events that arise both neurally and behaviorally in realistic social situations. 


\section{References}

1. Shteynberg, G., Hirsh, J. B., Bentley, R. A., \& Garthoff, J. (2020). Shared worlds and shared minds: A theory of collective learning and psychology of common knowledge. Psychological Review, 127, 918931. https://doi.org/10.1037/rev0000200.

2. Wolf, W., Launay, J., \& Dunbar, R. I. M. (2016). Joint attention, shared goals, and social bonding. British Journal of Psychology, 107(2), 322-337. https://doi.org/10.1111/bjop.12144.

3. Wolf, W. \& Tomasello, M. (2020). Watching a video together creates social closeness between children and adults. Journal of Experimental Child Psychology, 189, 104712.

https://doi.org/10.1016/j.jecp.2019.104712.

4. Shteynberg, G. (2015). Shared attention. Psychological Science, 10(5), 579-590. https://doi.org/10.1177/1745691615589104.

5. Stephenson, L. J., Edwards, S. G., \& Bayliss, A. P. (2021). From gaze perception to social cognition: The shared attention system. Perspectives on Psychological Science, Advanced Online Publication. https://doi.org/10.1177/1745691620953773.

6. Redcay, E., \& Saxe, R. (2013). Do you see what I see? The neural bases of joint attention. In J. Metcalfe \& H.S. Terrace, Eds., Agency and Joint Attention, 216-237. Oxford Scholarship Online. https://doi.org/10.1093/acprof:oso/9780199988341.001.0001.

7. Kingstone, A., Tipper, C., Ristic, J., \& Ngan, E. (2004). The eyes have it!: An fMRI investigation. Brain and Cognition, 55, 269-271. https://doi.org/10.1016/j.bandc.2004.02.037.

8. Posner, M. (1980). Orienting of Attention. Quarterly Journal of Experimental Psychology, 32, 3-25. https://doi.org/10.1080/00335558008248231.

9. Schilbach, L., Wilms, M., Eickhoff, S. B., Romanzetti, S., Tepest, R., Bente, G., Shah, N. J., Fink, G. R., \& Vogeley, K. (2010). Minds made for sharing: Initiating joint attention recruits reward-related circuitry. Journal of Cognitive Neuroscience, 22, 2702-2715. https://doi.org/10.1162/jocn.2009.21401.

10. Mundy, P. (2018). A review of joint attention and social-cognitive brain systems in typical development and autism spectrum disorder. European Journal of Neuroscience, 47, 497-514.

https://doi.org/10.1111/ejn.13720.

11. Bristow, D., Rees, G., \& Frith, C. D. (2007). Social interaction modifies neural response to gaze shifts. Social Cognitive and Affective Neuroscience, 2, 52-61. https://doi.org/10.1093/scan/ns1036.

12. Redcay, E., Dodell-Feder, D., Pearrow, M. J., Mavros, P. L., Kleiner, M., Gabrieli, J. D. E., \& Saxe, R. (2010). Live face-to-face interaction during fMRI: A new tool for social cognitive neuroscience. NeuroImage, 50, 1639-1647. https://doi.org/10.1016/j.neuroimage.2010.01.052. 
13. Bhanji, J. P., \& Delgado, M. R. (2014). The social brain and reward: Social information processing in the human striatum. Wiley Interdisciplinary Reviews: Cognitive Science, 5, 61-73.

https://doi.org/10.1002/wcs.1266.

14. Liu X., Hairston, J., Schrier, M., \& Fan, J. (2011). Common and distinct networks underlying reward valence and processing stages: A meta-analysis of functional neuroimaging studies. Neuroscience and Biobehavioral Reviews, 35, 1219-1236. https://doi.org/10.1016/j.neubiorev.2010.12.012.

15. Pfeiffer, U. J., Schilbach, L., Timmermans, B., Kuzmanovic, B., Georgescu, A. L., Bente, G., \& Vogeley, K. (2014). Why we interact: On the functional role of the striatum in the subjective experience of social interaction. NeuroImage, 101, 124-137. https://doi.org/10.1016/j.neuroimage.2014.06.061.

16. Rademacher, L., Krach, S., Kohls, G., Irmak, A., Gründer, G., \& Spreckelmeyer, K.N. (2010). Dissociation of neural networks for anticipation and consumption of monetary and social rewards. NeuroImage, 49, 3276-3285. https://doi.org/10.1016/j.neuroimage.2009.10.089.

17. Redcay, E., \& Moraczewski, D. (2019). Social cognition in context: A naturalistic imaging approach. NeuroImage, 216, 116392. https://doi.org/10.1016/j.neuroimage.2019.116392.

18. Zaki, J., \& Ochsner, K. (2009). The need for a cognitive neuroscience of naturalistic social cognition. Annual New York Academy of Sciences, 1167, 16-30. https://doi.org/10.1111/j.1749-6632.2009.04601.x.

19. Bartels, A., \& Zeki, S. (2003). The neural correlates of maternal and romantic love. NeuroImage, 21, 1155-1166. https://doi.org/10.1016/j.neuroimage.2003.11.003.

20. Fernández-Aguilar, L., Navarro-Bravo, B., Ricarte, J., Ros, L., Latorre, J. M. (2019). How effective are films in inducing positive and negative emotional states? A meta-analysis. PLoS ONE, 14, e0225040. https://doi.org/10.1371/journal.pone.0225040.

21. Golland, Y., Levit-Binnun, N., Hendler, T., \& Lerner, Y. (2017). Neural dynamics underlying emotional transmissions between individuals. Social Cognitive and Affective Neuroscience, 12, 1249-1260. https://doi.org/10.1093/scan/nsx049.

22. Haxby, J. V., Gobbini, M. I., \& Nastase, S. A. (2020). Naturalistic stimuli reveal a dominant role for agentic action in visual representation. NeuroImage, 216, 116561.

https://doi.org/10.1016/j.neuroimage.2020.116561.

23. Kikusui, T., Winslow, J. T., \& Mori, Y. (2006). Social buffering: Relief from stress and anxiety. Philosophical Transactions of the Royal Society B: Biological Sciences, 361, 2215-2228. https://doi.org/10.1098/rstb.2006.1941. 
24. Kiyokawa, Y., \& Hennessy, M. B. (2018). Comparative studies of social buffering: A consideration of approaches, terminology, and pitfalls. Neuroscience and Biobehavioral Reviews, 86, 131-141. https://doi.org/10.1016/j.neubiorev.2017.12.005.

25. Qi, Y., Hermann, M. J., Bell, L., Fackler, A., Han, S., Deckert, J., \& Hein, G. (2020). The mere physical presence of another person reduces human autonomic responses to aversive sounds. Proceedings of the Royal Society B, 287: 20192241. http://dx.doi.org/10.1098/rspb.2019.2241.

26. Wingenbach, T. S. H., Ribeiro, B., Nakao, C., Gruber, J., \& Boggio, P. S. (2019). Evaluations of affective stimuli modulated by another person's presence and affiliative touch. Emotion, 21, 360-375. Advance online publication. http://dx.doi.org/10.1037/emo0000700.

27. Beckes, L., \& Coan, J. A. (2011). Social baseline theory: The role of social proximity in emotion and economy of action. Social and Personality Psychology Compass, 5, 976-988.

http://doi.org/10.1111/j.1751-9004.2011.00400.x.

28. Apicella, C. L., Marlowe, F. W., Fowler, J. H., \& Christakis, N. A. (2012). Social networks and cooperation in hunter-gatherers. Nature, 481, 497-501. https://doi.org/10.1038/nature10736.

29. Axelrod, R. \& Hamilton, W. D. (1981). The Evolution of Cooperation. Science, 211, 1390-1396. https://doi.org/10.1126/science.7466396.

30. West, S. A., Griffin, A. S., \& Gardner, A. (2007) Evolutionary Explanations for Cooperation. Current Biology, 17, R661-R672. https://doi.org/10.1016/j.cub.2007.06.004.

31. Bugajski, J. (1999). Social stress adapts signaling pathways involved in stimulation of the hypothalamicpituitary-adrenal axis. Journal of Physiology and Pharmacology, 50, 367-379.

32. Gunnar, M. R. \& Hostinar, C. E. (2015). The social buffering of the hypothalamic-pituitaryadrenocortical axis in humans: Developmental and experiential determinants. Social Neuroscience, 10, 479-488. https://doi.org/10.1080/17470919.2015.1070747.

33. Koss, K. J., Hostinar, C. E., Donzella, B., \& Gunnar, M. R. (2014). Social deprivation and the HPA axis in early development. Psychoneuroendocrinology, 50, 1-13.

http://dx.doi.org/10.1016/j.psyneuen.2014.07.028.

34. Thomas, J. C., Letourneau, N., Campbell, T. S., Giesbrecht, G. F., \& Apron Study Team. (2018). Social buffering of the maternal and infant HPA axes: Mediation and moderation in the intergenerational transmission of adverse childhood experiences. Development and Psychopathology, 30, 921-939. https://doi.org/10.1017/S0954579418000512. 
35. Coan, J. A., Schaefer, H. S., \& Davidson, R. J. (2006). Lending a hand: Social regulation of the neural response to threat. Psychological Science, 17, 1032-1039. https://doi.org/10.1111/j.14679280.2006.01832.x.

36. Eisenberger, N. I., Taylor, S. E., Gable, S. L., Hilmert, C. J., \& Lieberman, M. D. (2007). Neural pathways link social support to attenuated neuroendocrine stress responses. NeuroImage, 35, 1601-1612. https://doi.org/10.1016/j.neuroimage.2007.01.038.

37. Parkinson, B. (2011). Interpersonal emotion transfer: Contagion and social appraisal. Social and Personality Psychology Compass, 5, 428-439. https://doi.org/10.1111/j.1751-9004.2011.00365.x.

38. Prochazkova, E. \& Kret, M. E. (2017). Connecting minds and sharing emotions through mimicry: A neurocognitive model of emotional contagion. Neuroscience and Biobehavioral Reviews, 80, 99-114. http://dx.doi.org/10.1016/j.neubiorev.2017.05.013.

39. Wróbel, M. \& Imbir, K. K. (2019). Broadening the perspective on emotional contagion and emotional mimicry: The correction hypothesis. Perspectives on Psychological Science, 14, 437-451. https://doi.org/10.1177/1745691618808523.

40. Páez, D., Rimé, B., Basabe, N., Wlodarczyk, A., \& Zumeta, L. (2015). Psychosocial effects of perceived emotional synchrony in collective gatherings. Journal of Personality and Social Psychology, 108, 711729. https://doi.org/10.1037/pspi0000014.

41. Shteynberg, G., Hirsh, J. B., Apfelbaum, E. P., Larsen, J. T., Galinsky, A. D., \& Roese, N. J. (2014). Feeling more together: Group attention intensifies emotion. Emotion, 14, 1102-1114. https://doi.org/10.1037/a0037697.

42. Wagner, U., Galli, L., Schott, B. H., Wold, A., van der Schalk, J., Manstead, A., Scherer, K., \& Walter, H. (2015). Beautiful friendship: Social sharing of emotions improves subjective feelings and activates the neural reward circuitry. Social Cognitive and Affective Neuroscience, 10, 801-808. https://doi.org/10.1093/scan/nsu121.

43. Nummenmaa, L., Glerean, E., Viinikainen, M., Jääskeläinen, I. P., Hari, R., Sams, M. (2012). Emotions promote social interaction by synchronizing brain activity across individuals. Proceedings of the National Academy of Sciences, Early Edition, 201206095. https://doi.org/10.1073/pnas.1206095109.

44. Hasson, U., Nir, Y., Levy, I., Fuhrmann, G., \& Malach, R. (2004). Intersubject synchronization of cortical activity during natural vision. Science, 303, 1634-1640. https://doi.org/10.1126/science.1089506.

45. Nastase, S. A., Gazzola, V., Hasson, U., \& Keysers, C. (2019). Measuring shared responses across subjects using intersubject correlation. Social Cognitive and Affective Neuroscience, 14, 667-685. https://doi.org/10.1093/scan/nsz037. 
46. Ben-Yakov, A., Honey, C. J., Lerner, Y., \& Hasson, U. (2012). Loss of reliable temporal structure in event-related averaging of naturalistic stimuli. NeuroImage, 63, 501-506.

https://doi.org/10.1016/j.neuroimage.2012.07.008.

47. Wilson, S. M., Molnar-Szakacs, I., \& Iacoboni, M. (2008). Beyond superior temporal cortex: Intersubject correlations in narrative speech comprehension. Cerebral Cortex, 18, 230-242. https://doi.org/10.1093/cercor/bhm049.

48. Peirce, J. W., Gray, J. R., Simpson, S., MacAskill, M. R., Höchenberger, R., Sogo, H., Kastman, E., Lindeløv, J. (2019). PsychoPy2: experiments in behavior made easy. Behavior Research Methods, 51, 195-203. https://doi.org/10.3758/s13428-018-01193-y.

49. Esteban, O., Markiewicz, C. J., Blair, R. W., Moodie, C. A., Isik, A. I., Erramuzpe, A., Kent, J. D., Goncalves, M., DuPre, E., Snyder, M., Oya, H., Ghosh, S. S., Wright, J., Durnez, J., Poldrack, R. A., \& Gorgolewski, K. J. (2019). fMRIPrep: a robust preprocessing pipeline for functional MRI. Nature Methods, 16(1), 111-116. https://doi.org/10.1038/s41592-018-0235-4.

50. Pruim, R. H. R., Mennes, M., Buitelaar, J. K., \& Beckmann, C. F. (2015). Evaluation of ICA-AROMA and alternative strategies for motion artifact removal in resting state fMRI. NeuroImage, 112, 278-287. https://doi.org/10.1016/j.neuroimage.2015.02.063.

51. Chen, G., Taylor, P. A., Shin, Y.-W., Reynolds, R. C., \& Cox, R. W. (2017). Untangling the relatedness among correlations, part II: Inter-subject correlation group analysis through linear mixed-effects modeling. NeuroImage, 147, 825-840. https://doi.org/10.1016/j.neuroimage.2016.08.029.

52. Moraczewski, D., Chen, G., \& Redcay, E. (2018). Inter-subject synchrony as an index of functional specialization in early childhood. Scientific Reports, 8, 2252. https://doi.org/10.1038/s41598-018-206000 .

53. Moraczewski, D., Nketia, J., \& Redcay, E. (2020). Cortical temporal hierarchy is immature in middle childhood. NeuroImage, 2016, 116616. https://doi.org/10.1016/j.neuroimage.2020.116616.

54. Yarkoni, T., Poldrack, R. A., Nichols, T. E., Van Essen, D. C., \& Wager, T. D. (2012). Large-scale automated synthesis of human functional neuroimaging data. Nature Methods, 8, 665-70. https://doi.org/10.1038/nmeth.1635.

55. Jolly, E., Tamir, D. I., Burum, B., \& Mitchell, J. P. (2019). Wanting without enjoying: The social value of sharing experiences. PLoS ONE, 14, e0215318. https://doi.org/10.1371/journal.pone.0215318.

56. Williams, J. H. G., Waiter, G. D., Perra, O., Perrett, D. I., \& Whiten, A. (2005). An fMRI study of joint attention experience. NeuroImage, 25, 133-140. https://doi.org/10.1016/j.neuroimage.2004.10.047. 
57. Caruana, N., McArthur, G., Woolgar, A., \& Brock, J. (2015). Simulating social interactions for the experimental investigation of joint attention. Neuroscience and Biobehavioral Reviews, 74, 115-125. http://dx.doi.org/10.1016/j.neubiorev.2016.12.022.

58. Redcay, E., Kleiner, M., \& Saxe, R. (2012). Look at this: The neural correlates of initiating and responding to bids for joint attention. Frontiers in Human Neuroscience, 6, 169.

https://doi.org/10.3389/fnhum.2012.00169.

59. Mundy, P. \& Newell, L. (2007). Attention, joint attention, and social cognition. Current Directions in Psychological Science, 16, 269-274. https://doi.org/10.1111/j.1467-8721.2007.00518.x.

60. Pfeiffer, U. J., Schilbach, L., Timmermans, B., Kuzmanovic, B., Georgescue, A. L., Bente, G., \& Vogeley, K. (2014). Why we interact: On the functional role of the striatum in the subjective experience of social interaction. NeuroImage, 101, 124-137. http://dx.doi.org/10.1016/j.neuroimage.2014.06.061.

61. Schilbach, L., Bzdok, D., Timmermans, B., Fox, P. T., Laird, A. R., Vogeley, K., \& Eickhoff, S. B. (2012). Introspective minds: Using ALE meta-analyses to study commonalities in the neural correlates of emotional processing, social \& unconstrained cognition. PLOS ONE, 7, e30920. https://doi.org/10.1371/journal.pone.0030920.

62. Welborn, B. L. \& Lieberman, M. D. (2014). Person-specific theory of mind in medial pFC. Journal of Cognitive Neuroscience, 27, 1-12.https://doi.org/10.1162/jocn_a_00700.

63. Saxe, R. (2006). Uniquely human social cognition. Current Opinion in Neurobiology, 16, 235-239. https://doi.org/10.1016/j.conb.2006.03.001.

64. Amft, M., Bzdok, D., Laird, A. R., Fox, P. T., Schilbach, L., \& Eickhoff, S. B. (2015). Definition and characterization of an extended social-affective default network. Brain Structure and Function, 220, 1031-1049. https://doi.org/10.1007/s00429-013-0698-0.

65. Bzdok, D., Langner, R., Schilbach, L., Engermann, D. A., Laird, A. R., Fox, P. T., \& Eickhoff, S. B. (2013). Segregation of the human medial prefrontal cortex in social cognition. Frontiers in Human Neuroscience, 7, 232. https://doi.org/10.3389/fnhum.2013.00232.

66. Daniel, R. \& Pollman, S. (2014). A universal role of the ventral striatum in reward-based learning: evidence from human studies. Neurobiology of Learning and Memory, 114, 90-100. https://doi.org/10.1016/j.nlm.2014.05.002.

67. Diekhof, E. K., Kaps, L., Falkai, P., \& Gruber, O. (2012). The role of the human ventral striatum and the medial orbitofrontal cortex in the representation of reward magnitude - an activation likelihood estimation meta-analysis of neuroimaging studies of passive reward expectancy and outcome processing. Neuropsychologia, 50, 1252-1266. https://doi.org/10.1016/j.neuropsychologia.2012.02.007. 
68. Amodio, D. M. \& Frith, C. D. (2006). Meeting of minds: The medial frontal cortex and social cognition. Nature Reviews Neuroscience, 7, 268-277. https://doi.org/10.1038/nrn1884.

69. Olsson, A. \& Ochsner, K. N. (2008). The role of social cognition in emotion. Trends in Cognitive Neuroscience, 12, 65-71. https://doi.org/10.1016/j.tics.2007.11.010.

70. Van Overwalle, F. (2009). Social cognition and the brain: A meta-analysis. Human Brain Mapping, 30 , 829-858. https://doi.org/10.1002/hbm.20547.

71. Konu, D., Turnbull, A., Karapanagiotidis, T., Wang, H.-T., Brown, L. R., Jefferies, E., \& Smallwood, J. (2020). A role for the ventromedial prefrontal cortex in self-generated episodic social cognition. NeuroImage, 218, 116977. https://doi.org/10.1016/j.neuroimage.2020.116977.

72. Adolphs, R. (2009). The social brain: Neural basis of social knowledge. Annual Review of Psychology, 60, 693-716. https://doi.org/10.1146/annurev.psych.60.110707.163514.

73. Phelps, E. A. \& LeDoux, J. E. (2005). Contributions of the amygdala to emotion processing: from animal models to human behavior. Neuron, 48, 175-187. https://doi.org/10.1016/j.neuron.2005.09.025.

74. Fox, A. S., Oler, J. A., Tromp, D. P. M., Fudge, J. L., \& Kalin, N. H. (2015). Extending the amygdala in theories of threat processing. Trends in Neurosciences, 38, 319-329.

https://doi.org/10.1016/j.tins.2015.03.002.

75. Vuilleumier, P. (2005). How brains beware: Neural mechanisms of emotional attention. Trends in Cognitive Sciences, 9, 585-594. https://doi.org/10.1016/j.tics.2005.10.011.

76. Bhanji, J. \& Delgado, M. R. (2014). The social brain and reward: Social information processing in the human striatum. Wiley Interdisciplinary Reviews: Cognitive Science, 5, 61-73. https://doi.org/10.1002/wcs.1266.

77. Fareri, D. S. \& Delgado, M. R. (2014). Differential reward responses during competition against in- and out-of-network others. Social Cognitive and Affective Neuroscience, 9, 412-420. https://doi.org/10.1093/scan/nst006.

78. Rilling, J., Gutman, D., Zeh, T., Pagnoni, G., Berns, G., \& Kilts, C. (2002). A neural basis for social cooperation. Neuron, 35, 395-405. https://doi.org/10.1016/s0896-6273(02)00755-9.

79. Fredrickson, B. L. \& Joiner, T. (2002). Positive emotions trigger upward spirals toward emotional wellbeing. Psychological Science, 13, 172-175. https://doi.org/10.1111/1467-9280.00431.

80. Arewasikporn, A., Sturgeon, J. A., Zautra, A. J. (2019). Sharing positive experiences boosts resilient thinking: Everyday benefits of social connection and positive emotion in a community sample. American Journal of Community Psychology, 63, 110-121. https://doi.org/10.1002/ajcp.12279. 
81. Pearce, E., Launay, J., \& Dunbar, R. I. M. (2015). The ice-breaker effect: Singing mediates fast social bonding. Royal Society Open Science, 2, 150221. https://doi.org/10.1098/rsos.150221.

82. Tarr, B., Launay, J., \& Dunbar, R. I. M. (2015). Silent disco: Dancing in synchrony leads to elevated pain thresholds and social closeness. Evolution and Human Behavior, 37, 343-349. https://doi.org/10.1016/j.evolhumbehav.2016.02.004.

83. Arsalidou, M., Duerden, E. G., \& Taylor, M. J. (2013). The centre of the brain: Topographical model of motor, cognitive, affect, and somatosensory functions of the basal ganglia. Human Brain Mapping, 34, 3031-3054. https://doi.org/10.1002/hbm.22124.

84. Harsay, H. A., Cohen, M. X., Oosterhof, N. N., Forstmann, B. U., Mars, R. B., \& Ridderinkhof, K. R. (2011). Functional connectivity of the striatum links motivation to action control in humans. The Journal of Neuroscience, 31, 10701-10711. https://doi.org/10.1523/JNEUROSCI.5415-10.2011.

85. Hasson, U., Malach, R., \& Heeger, D. J. (2010). Reliability of cortical activity during natural stimulation. Trends in Cognitive Sciences, 14, 40-48. https://doi.org/10.1016/j.tics.2009.10.011.

86. Hasson, U., Yang, E., Vallines, I., Heeger, D. J., \& Rubin, N. (2008). A hierarchy of temporal receptive windows in human cortex. The Journal of Neuroscience, 28, 2539-2550. https://doi.org/10.1523/JNEUROSCI.5487-07.2008.

87. Lerner, Y., Honey, C. J., Silbert, L. J., \& Hasson, U. (2011). Topographic mapping of a hierarchy of temporal receptive windows using a narrated story. The Journal of Neuroscience, 31, 2906-2915. https://doi.org/10.1523/JNEUROSCI.3684-10.2011.

88. Brewer, A. A., Liu, J., Wade, A. R., \& Wandell, B. A. (2005). Visual field maps and stimulus selectivity in human ventral occipital cortex. Nature Neuroscience, 8, 1102-1109. https://doi.org/10.1038/nn1507.

89. Nelken, I. (2004). Processing of complex stimuli and natural scenes in the auditory cortex. Current Opinion in Neurobiology, 14, 474-480. https://doi.org/10.1016/j.conb.2004.06.005.

90. Hasson, U., Chen, J., \& Honey, C. J. (2015). Hierarchical process memory: Memory as an integral component of information processing. Trends in Cognitive Sciences, 19, 304-313. http://dx.doi.org/10.1016/j.tics.2015.04.006.

91. Honey, C. J., Thesen, T., Donner, T. H., Silbert, L. J., Carlson, C. E., Devinsky, O., Doyle, W. K., Rubin, N., Heeger, D. J., Hasson, U. (2012). Slow cortical dynamics and the accumulation of information over long timescales. Neuron, 76, 423-434. http://dx.doi.org/10.1016/j.neuron.2012.08.011. 